\title{
BMJ Open What stage are low-income and middle- income countries (LMICs) at with patient safety curriculum implementation and what are the barriers to implementation? A two-stage cross-sectional study
}

\author{
Liane R Ginsburg, ${ }^{1}$ Neelam Dhingra-Kumar, ${ }^{2}$ Liam J Donaldson ${ }^{3}$
}

To cite: Ginsburg LR, DhingraKumar N, Donaldson LJ. What stage are low-income and middle-income countries (LMICs) at with patient safety curriculum implementation and what are the barriers to implementation? A two-stage cross-sectional study. BMJ Open 2017;7:e016110. doi:10.1136/ bmjopen-2017-016110

Received 26 January 2017 Revised 18 April 2017 Accepted 11 May 2017

\section{CrossMark}

${ }^{1}$ Health Policy \& Management, York University, Toronto, Ontario, Canada

${ }^{2}$ Service Delivery and Safety, World Health Organization, Geneva, Switzerland ${ }^{3}$ London School of Hygiene and Tropical Medicine, NonCommunicable Disease Epidemiology, London, UK

Correspondence to Dr Liane R Ginsburg; Igins@ yorku.ca

\section{ABSTRACT}

Objectives The improvement of safety in healthcare worldwide depends in part on the knowledge, skills and attitudes of staff providing care. Greater patient safety content in health professional education and training programmes has been advocated internationally. While WHO Patient Safety Curriculum Guides (for Medical Schools and Multi-Professional Curricula) have been widely disseminated in low-income and middle-income countries (LMICs) over the last several years, little is known about patient safety curriculum implementation beyond high-income countries. The present study examines patient safety curriculum implementation in LMICs.

Methods Two cross-sectional surveys were carried out. First, 88 technical officers in Ministries of Health and WHO country offices were surveyed to identify the pattern of patient safety curricula at country level. A second survey followed that gathered information from 71 people in a position to provide institution-level perspectives on patient safety curriculum implementation.

Results The majority, 69\% (30/44), of the countries were either considering whether to implement a patient safety curriculum or actively planning, rather than actually implementing, or embedding one. Most organisations recognised the need for patient safety education and training and felt a safety curriculum was compatible with the values of their organisation; however, important faculty-level barriers to patient safety curriculum implementation were identified. Key structural markers, such as dedicated financial resources and relevant assessment tools to evaluate trainees' patient safety knowledge and skills, were in place in fewer than half of organisations studied.

Conclusions Greater attention to patient safety curriculum implementation is needed. The barriers to patient safety curriculum implementation we identified in LMICs are not unique to these regions. We propose a framework to act as a global standard for patient safety curriculum implementation. Educating leaders through the system in order to embed patient safety culture in education and clinical settings is a critical first step.
Strengths and limitations of this study

- WHO Patient Safety Curriculum Guides (for Medical Schools and Multi-Professional Curricula) have been widely disseminated in low-income and middleincome countries (LMICs) starting in 2009 in an effort to help address the global problem of patient safety; this study provides the first multicountry assessment of patient safety curriculum implementation beyond high-income nations; the inquiry is theory driven.

- The present paper proposes a theory-based framework that can act as a global standard for patient safety curriculum implementation and may also be useful when other substantial curricular shifts in health professional education and training are undertaken.

- Although follow-up data from non-responders suggests our overall results may overestimate, rather than underestimate, the state of patient safety curricular implementation in LMICs, our results are based on a small respondent group and we are unable to comment on differences in implementation between LMICs.

- The African region has the highest proportion of lowincome countries, and we were unable to include countries in this region; the four regions that did participate in this study also showed large variation in the proportion of countries able to identify a patient safety focal point. Variation in patient safety curriculum implementation across LMICs is an area that requires further study.

\section{INTRODUCTION}

Several key policy groups internationally have advocated the need for greater patient safety content in health professional education and training programmes. ${ }^{1-7}$ In response, some well-developed patient safety science curricula have been created for medical and other health professional students and 
postgraduates. ${ }^{89}$ Such programmes seek to teach about the system factors, the human factors, patient engagement and other sociocultural factors that contribute to patient safety in particular and safety in healthcare in general and to address harmful aspects of the underlying culture found in the hidden curriculum. ${ }^{10}$ Achieving reform in health professional education is difficult and evidence points to patient safety science being incorporated into curricula slowly and unevenly. ${ }^{11-13}$ Numerous local intervention studies have evaluated the impact of a patient safety curriculum on medical students' safety knowledge and/or safety attitudes. ${ }^{14-21}$ Most of these studies have been confined to a single educational institution. Questions remain regarding which stage of health professional training is most appropriate for learning about patient safety. ${ }^{22}$ Patient safety curriculum implementation is further complicated by the need for patient safety to be integrated into all aspects of health professional education and training-stand-alone patient safety courses are not what is needed. ${ }^{23}$

From a global perspective, while patient safety curricular resources have been developed for high-income as well as low-income and middle-income countries and specific low-resource educational settings, ${ }^{8}$ merely making these resources available is unlikely to lead to curricular change. ${ }^{24-26}$ Greater focus is needed in low-income, middle-income and high-income countries regarding the best ways to implement patient safety curricula. Indeed, research in high-income countries suggests that as few as one quarter of medical schools formally teach patient safety ${ }^{13}$ and such teaching typically involved 1-2 days of teaching ${ }^{11}$ delivered in the form of short courses or modules. ${ }^{27}$ Others have highlighted the need for greater progress integrating patient safety in health professional education. $^{28}$

In low-income and middle-income countries, little is known about the extent of use of patient safety curricula: what topics are included, what stage are countries at with patient safety curriculum implementation and what are the barriers to implementation. To address these gaps in knowledge, the present study looks at patient safety curriculum implementation in low-income and middle-income countries. We examined curriculum implementation using a four-stage framework derived from the field of implementation science ${ }^{25} 29$ as well as literature on organisational readiness for curriculum change, ${ }^{30}$ successful curricular change in medical schools ${ }^{31}$ and research on facilitators and barriers to nationwide implementation of a new postgraduate medical curriculum. ${ }^{32}$ Because the current study focuses on low-income and middle-income countries, research on medical school curricular reform in these settings ${ }^{33}$ was also used to ensure we examined curriculum implementation stages and barriers germane to the low-income and middle-income country context.

\section{METHODS}

The study design comprised two cross-sectional surveys.

\section{Study populations}

WHO Member States are grouped into six regions; each region has a regional office. WHO has more than 150 country offices, mostly located in low-income and middle-income countries. ${ }^{34} 35$ The first study population was those technical officers with responsibilities for patient safety and quality programmes working in WHO country offices and Ministries of Health; it comprised 88 people from 62 low-income and middle-income countries in four of six WHO regions: Southeast Asia, Western Pacific, Eastern Mediterranean and the Americas. These 88 individuals were identified by the director responsible for service delivery in each region. The WHO African and European Regions could not be included because of changes in leadership and privacy policies, respectively.

The first study period was the month of January 2016. The purpose of this first country-level survey was to obtain information on patient safety curriculum implementation in countries and to identify people with in-depth knowledge of health professional education and training to be the study population for the second survey.

The second study population comprised 71 people (representing 26 countries), identified by the WHO and Ministry of Health technical officers in the first survey, who were judged to have in-depth knowledge of health professional education and training. These individuals were from regional or national health professional education councils and educational bodies who had been involved in and/or were aware of patient safety curriculum implementation activities.

The second study period was the month of April 2016. The purpose of this within-country survey was to gather more detailed data about patient safety curriculum implementation.

In both surveys, we obtained information from members of the study populations through an anonymous online form available through a link embedded in an email invitation (limesurvey.org). We sent two follow-up reminders at 10-day intervals.

\section{Measures}

For the country-level survey, we defined four stages of curriculum implementation (see box 1). We asked respondents to choose the implementation stage that best described the overall situation in their country.

In the second within-country survey, we asked respondents whether their organisation was aware of any patient safety curricula and to judge the status of implementation (decided not to implement, in the process of deciding and proceeding with implementation). We then asked them to report, using a 5-point agreement Likert scale, on 21 items reflecting key activities and markers at each stage of implementation. In addition, we requested responses on 23 binary variables that reflected barriers to curriculum implementation related to: the curriculum itself, the context or setting and the implementation process. We chose barriers that were widely cited in the implementation science literature. 
Box 1 Four stages of curriculum implementation

Stage 1: considering the need for the curriculum The organisation is deciding whether to adopt a new patient safety curriculum. They may consider whether it fits with the values of the organisation, whether it is feasible to implement and so on.

\section{Stage 2: planning for implementation}

For example, identifying those who will lead the process, committing resources, introducing the curriculum to faculty, setting implementation timelines.

\section{Stage 3: delivering the new curriculum}

For example, teaching new patient safety modules, involving teachers in clinical settings, perhaps evaluating the implementation process.

\section{Stage 4: embedding the new curriculum}

Patient safety curriculum and its values are embedded throughout the health professional education process.

Both surveys were web based, devised by the research team and defined implementation items and barriers using existing theory and empirical work on curriculum implementation and work from the field of implementation science. We pilot-tested the survey for clarity and modified it as a result. Descriptive statistics are presented.

\section{Ethical approval}

The Research Ethics Board of the first author's home institution and the WHO Research Ethics Review Committee both approved the study.

\section{RESULTS}

In the first survey, we made contact with 85 individuals in 62 countries (email addresses for 3 of the 88 individuals in the first study population were inaccurate and could not be corrected). Fifty-two per cent of them (44/85) participated, representing $44 \%(27 / 62)$ of countries. Not all first survey respondents identified people to receive the second survey.

Respondents in the first survey identified 71 people in 26 countries whom they felt had sufficiently detailed knowledge about implementation of patient safety curriculum to participate in the second survey. Of these, $42 \%$ (30/71) responded to the second survey. An additional response was received from a contact in one middle-income country in Europe for a total of 31 usable responses on the second survey. The number of countries represented by these 31 individuals is not known as one quarter of these respondents did not indicate which country they represent. Forty-two per cent (13/31) of respondents worked in universities, $42 \%(13 / 31)$ in ministries of health and $7 \%(2 / 31)$ in health professional councils.

\section{Patient safety curriculum implementation}

Overall, the majority, $69 \%(30 / 44)$, of countries were either considering whether to implement a patient safety curriculum or actively planning for implementation (table 1). Those only considering implementation were deliberating on such factors as whether a proposed new curriculum would fit with the values of the organisation or be feasible to implement. Those actively planning introduction were considering who would lead the process, introducing the curriculum to faculty and establishing timelines. In 9\% (4/44) of countries, respondents reported that a patient safety curriculum was embedded throughout the health professional education process. We have few details for these four countries-only two countries returned the second survey and both identified several barriers to implementation.

The second survey, in which information was sought and received from knowledgeable individuals within countries, yielded a similar distribution of status of implementation; the difference was that respondents at this level were not asked to specify an overall implementation stage (table 1).

We followed up with non-responders to both surveys to assess non-response bias. We heard back from $25 \%$ of these non-responders and most stated that they did not return a survey because they were not aware of any patient safety curriculum work going on. The next most common reasons for non-response were that the organisation was in the midst of deciding whether or how to implement a patient safety curriculum (so they felt the survey did not apply to them yet), or that they could not respond for technical reasons (eg, the survey invitation went to spam or the electronic link would not work).

Table 1 Overall stage of patient safety curriculum implementation

\begin{tabular}{lll} 
Stage of patient safety curriculum implementation & $\begin{array}{l}\text { Country-level survey } \\
\%(\mathbf{n}) \mathbf{n = 4 4}\end{array}$ & $\begin{array}{l}\text { Within- country survey } \\
\%(\mathbf{n}) \mathbf{n = 3 1}\end{array}$ \\
\hline $\begin{array}{l}\text { Not aware of/have not considered implementation of any } \\
\text { patient safety curriculum }\end{array}$ & $11(5)$ & $13(4)$ \\
Decided not to implement now & $\mathrm{n} / \mathrm{a}$ & $13(4)$ \\
Stage 1: considering the need for the curriculum & $39(17)$ & $55(17)$ \\
Stage 2: planning for implementation & $30(13)$ & $19(6)$ \\
Stage 3: delivering the new curriculum & $11(5)$ & $9(4)$ \\
Stage 4: embedding the new curriculum & &
\end{tabular}


We considered whether the PS Curriculum is aligned with the mission and goals of the organisation

We considered whether there is a NEED to implement the PS Curriculum

We considered whether implementation of the PS Curriculum would improve health professional education We considered if the values upon which the PS Curriculum is based are compatible with the values of ... We have buy-in from influential external organisations around the change

We have buy-in from senior management around the change

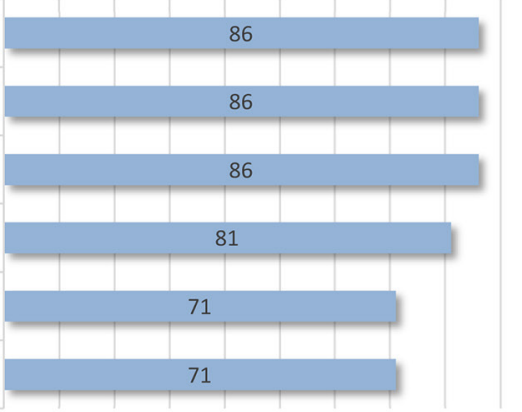

Initiating / Planning for Curricular Change (N=17)

An individual or team with the authority to implement the PS Curriculum has taken a leadership role in the change... Faculty training needs have been identified (so the PS Curriculum can be delivered)

The PS Curriculum has been widely shared with faculty who are likely to teach the curriculum

Clear roles and responsibilities for implementation have been defined

Clear timelines for implementation have been defined

New evaluation tools have been developed to assess student learning about aspects of patient safety covered Funds have been provided directly to support this initiative

Faculty have received high quality training in the areas covered by the PS Curriculum

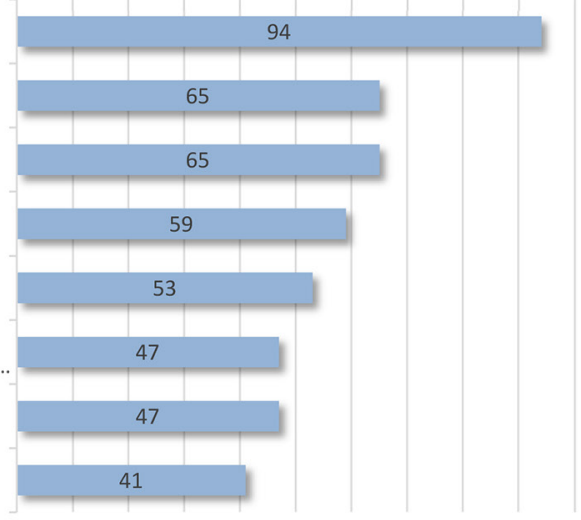

Patient Safety Curriculum Implementation ( $N=17$ )

The PS Curriculum is being widely delivered to students by faculty

A "systems" approach to PS, including ideas about fallibility, are now part of most aspects of HP education Faculty in clinical settings implement the principles of the PS Curriculum as they supervise trainees

The PS Curriculum is being implemented in its original (few major changes to the material have been made) Adjustments to the curriculum implementation process have been made in response to feedback

Formal evaluation of the PS Curriculum has been carried out

Informal feedback on implementation of the PS Curriculum is routinely gathered
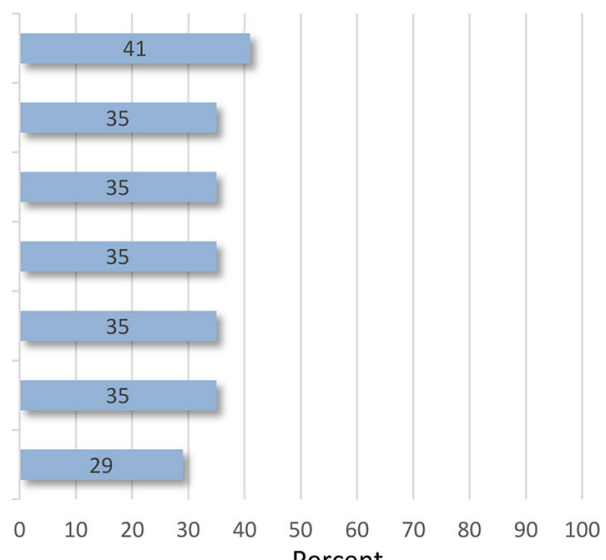

Figure 1 PS curriculum implementation activities by stage. PS, patient safety.

\section{Curriculum implementation activities}

In the second survey, $43 \%$ of respondents $(9 / 21)$ reported implementing one of the WHO patient safety curriculum guides, 29\% (6/21) were implementing a patient safety curriculum adapted from one of the WHO guides and $29 \%(6 / 21)$ did not name the patient safety curriculum they were implementing. Respondents provided detailed data on key markers and activities at each implementation stage (figure 1). For the first stage, 'Considering curricular change activities', agreement was highest on markers that reflected the perceived need for a patient safety curriculum and the fit of the curriculum with the mission and values of the organisation. Agreement was lower on having buy-in from senior management and influential external organisations. For the second stage of curriculum implementation, 'Initiating / planning for curricular change', nearly all respondents who completed this section of the survey $(94 \% ; 16 / 17)$ agreed that an individual or team with the authority to implement the curriculum had taken a leadership role in the change process and $65 \%(11 / 17)$ agreed that activities such as widely sharing the patient safety curriculum with faculty and identifying teacher training needs had taken place. Fewer than half of respondents agreed that 'Faculty received high quality training in the areas covered by the Patient Safety Curriculum' or that 'Funds were provided directly to support this initiative'. For the third and fourth stages, 'Implementing/institutionalising patient safety curricular change', data suggest that approximately $35 \%-40 \%$ of organisations had widely delivered, formally 
evaluated and adjusted the curriculum, that faculty in clinical settings implement patient safety principles and that a systems approach to patient safety, including ideas about fallibility, was part of all or nearly all aspects of health professional education. One item that reflects fidelity of patient safety curriculum implementation shows that $35 \%$ of organisations were implementing a patient safety curriculum in its original form.

Of the 18 respondents implementing a patient safety curriculum, fewer than half indicated that safety science topics such as human factors $(7 / 17)$, understanding systems $(7 / 17)$ and other non-technical skills such as being an effective team player $(3 / 17)$ had been taught while at least half indicated that medication safety $(10 / 17)$ and 'what is patient safety' $(12 / 17)$ had been taught. About half the respondents provided free text comments suggesting that implementation took the form of brief patient safety modules or lectures or was covered in existing courses on clinical safety.

Barriers to patient safety curriculum implementation

Barriers to patient safety curriculum implementation were captured in three categories: barriers pertaining to the patient safety curriculum itself, barriers related to the context for implementation and barriers related to the process of patient safety curriculum implementation (table 2). For barriers related to the curriculum itself, 24\% (5/21) of respondents identified 'Belief that the patient safety Curriculum was externally imposed' as a barrier. Other barriers pertaining to the curriculum

Table 2 Barriers to patient safety curriculum implementation

\section{$\%$ (n) identifying \\ as a barrier}

$\mathrm{n}=\mathbf{2 1}$

$10(2)$

$10(2)$

$19(4)$

$24(5)$

\section{Potential barriers related to the curriculum itself}

Belief by leadership that the patient safety curriculum is NOT implementable.

Belief by leadership that the patient safety curriculum will NOT improve health professional education. Belief by leadership that the patient safety curriculum is NOT needed.

Belief by leadership that the patient safety curriculum is externally imposed on us and not relevant to our context.

\section{Potential barriers related to the context}

38 (8)

$19(4)$

38 (8)

$43(9)$

$14(3)$

$33(7)$
Lack of buy-in from stakeholders internal to the organisation. Lack of buy-in from stakeholders external to the organisation.

Poor fit between the patient safety curriculum and the broader political and economic context (such as regulation of health professionals and how faculty are paid).

Lack of governmental commitment to the patient safety curriculum (eg, providing ongoing financial support).

Lack of organisation-level commitment to implementation of the patient safety curriculum.

Belief that the patient safety curriculum is NOT compatible with the values of the organisation (eg, the system view of safety, which recognises that clinicians make mistakes, is widely shared by leaders and educators across the organisation).

Poor fit between the patient safety curriculum and the assessment system in training settings (eg, the university).

24 (5) There is no space in the curriculum to add new content.

\section{Potential barriers related to the implementation process}

$67(14)$ Insufficient training to enable faculty to implement the patient safety curriculum.

$33(7)$ Poor coordination between the ministry and other organisations around implementation of the patient safety curriculum.

\section{Lack of faculty enthusiasm/meaningful participation in implementation of the curriculum.}

Poor communication channels among stakeholders regarding implementation of the curriculum. Implementation plan was not made clear to the implementers. Lack of faculty cooperation and collaboration to address implementation challenges. Insufficient evaluation of the curriculum implementation process. Lack of an influential person leading implementation of the patient safety curriculum. Inappropriate leadership approach to implementing the patient safety curriculum. Change(s) in leadership around the time of implementation of the patient safety curriculum. No clear role for teachers in clinical settings in the curriculum implementation process 
itself were identified by fewer than $20 \%$ (5) of respondents.

For barriers related to the implementation context, at least one-third of respondents identified several contextual barriers external to the organisation including lack of governmental commitment to the patient safety curriculum $(43 \%$; 9/21) and poor fit between the curriculum and aspects of the broader political and economic context such as remuneration systems $(38 \% ; 8 / 21)$. A similar number identified barriers in the internal context including lack of commitment from senior leadership $(43 \% ; 9 / 21)$, lack of buy-in from internal stakeholders $(38 \% ; 8 / 21)$ and poor fit between the patient safety curriculum and the system for assessing health professional trainees $(33 \% ; 7 / 21)$. Compatibility of the patient safety curriculum with the values of the organisation was identified as a barrier by a small number of respondents $(14 \% ; 3 / 21)$.

Finally, for barriers related to the implementation process, relatively few respondents $(14 \% ; 3 / 21)$ identified barriers related to communication of curricular changes. Thirty per cent of respondents identified faculty-level barriers including lack of enthusiasm $(7 / 21)$, lack of cooperation and collaboration to address implementation challenges $(7 / 21)$ and lack of a clear role for teachers in clinical settings $(6 / 21)$. The most frequently identified barrier to patient safety curriculum implementation was 'insufficient training to enable faculty to implement the Patient Safety Curriculum', which was identified by $67 \%$ of respondents $(14 / 21)$.

\section{DISCUSSION}

Our study contributes to knowledge about the extent of patient safety curriculum implementation in low-income and middle-income countries. We found that the majority of organisations were at the consideration or planning stages of implementation rather than the delivering and embedding stages. Most commonly identified barriers to curriculum implementation concerned faculty-level barriers including lack of cooperation and collaboration to address implementation challenges, absence of a clear role for clinical supervisors in practice settings and insufficient training to enable implementation of a patient safety curriculum (the latter barrier was identified by two-thirds of respondents). Lack of governmental commitment to the patient safety curriculum (eg, providing ongoing financial support) was the largest contextual barrier to implementation that was identified. Lack of commitment from senior leadership and low levels of buy-in from other internal stakeholders as well as poor fit between the patient safety curriculum and systems for faculty remuneration and trainee assessment were also identified as barriers by approximately one-third of respondents.

The early stage of patient safety curriculum implementation is consistent with the knowing-doing gap in a wide range of fields. ${ }^{36}$ Rather than create a patient safety curriculum and allow it to diffuse, organisations need help to make it happen. ${ }^{37}$ This is true for low-income and middle-income countries, as well as high-income countries; for example, research suggests that as few as one quarter of North American medical schools formally teach patient safety ${ }^{13}$ and such teaching typically involved 10 or fewer contact hours. ${ }^{11}$

The consensus statement from the 2009 Millennium Conference on Teaching Patient Safety in the 21 st century suggests that lack of guidance on how to implement, integrate and evaluate new curricular material on the foundations of patient safety science accounts for the low rate of uptake of patient safety curricula in health professional education and training. ${ }^{38}$

The ultimate goal for patient safety education is to integrate patient safety science and patient safety competencies throughout the system of health professional education. ${ }^{138-41}$ The approach must move away from the pockets of patient safety education (eg, stand-alone modules or courses) that currently dominate. ${ }^{42} 43$ It needs a shift towards embedding patient safety across all areas of education, including clinical teaching settings, ${ }^{40}$ where the kind of situated learning necessary to develop competence in teamwork, patient engagement and other non-technical skills takes place. ${ }^{27}$ As Walton and Barraclough point out, achieving major change in patient safety curriculum implementation will be very difficult without the engagement of clinical supervisors who can demonstrate and impart a patient safety mindset at the bedside. ${ }^{44}$ Embedding a patient safety curriculum is very difficult. In addition to the concrete implementation barriers we identified, sustaining a patient safety educational programme also requires changing the culture in academic medical faculties. There, strong views are held on the appropriateness of topics for educating the doctors of the future. The legitimacy of quality and safety concepts often requires strong justification when measured against the bioscientific model of practice that is traditionally valued. ${ }^{45}$

The findings of our study point to three key actions to help embed patient safety as a core topic for health professional learners. ${ }^{45}$

First, there is a need to build leadership capacity. We found that most organisations were in the early stages (adoption decision or initial planning). Strong leaders are able to establish a compelling need for change, convey a sense of urgency and build an implementation plan. Indeed, we suggest that leadership in patient safety curriculum implementation may be the most vital step. However, leaders themselves need to receive foundational education in patient safety science. ${ }^{146}$ This includes leaders in university settings, clinical training environments (ie, hospitals and clinics), educational accreditation and licensure bodies and those with administrative or policy functions that have a bearing on funding. Four of the 12 recommendations from the Lucian Leape Institute Expert Roundtable on Medical Education Reform ${ }^{1}$ concern creating learning cultures that promote and build capacity in foundational areas 
of patient safety competence, including professionalism, humility and other non-technical skills. The recommendations are clear that this capacity needs to be developed first among medical education leaders. The need for academic medical leadership to enhance legitimacy of patient safety research and training has been underscored by others. ${ }^{45}{ }^{47}$ Building broad leadership for patient safety is necessary for embedding a culture of safety and for ensuring the attitudes of existing clinicians and practices are able to embrace rather than reject incoming graduates of a new patient safety science-based curriculum. For medical education leaders in low-income and middle-income countries where there may be poor educational infrastructure, little patient safety technical content available or few human and financial resources to teach patient safety, the WHO Patient Safety Curriculum Guides (for Medical Schools ${ }^{48}$ and Multi-Professional Education $)^{49}$ can be a valuable resource,$^{50}$ providing practical support and guidance on what patient safety content to deliver and how to integrate it into existing curricula consistent with the local environment. ${ }^{28}$

Second, action must be directed towards the curriculum itself. Patient safety education should focus on safety science (human factors, systems, safety culture and patient engagement) and other non-technical skills. Key work on curriculum design ${ }^{51}$ emphasises the need to adapt to local training contexts. ${ }^{32}$ Regional and national differences in how health professional education is organised and governed are a key dimension of this context, along with factors such as: the resource richness of a setting, the way faculty are remunerated and ensuring that key stakeholders' needs are met.

Development and implementation of assessment systems to measure patient safety knowledge and skills are also essential components of the patient safety curriculum implementation process, as is evaluation more generally.

Thirdly, there is a need to leverage change at the health system level by including patient safety in undergraduate and graduate medical accrediting body competencies and programme requirements. For instance, the Australian Medical Council and the United Kingdom General Medical Council outline patient safety competencies and require institutions to demonstrate that patient safety learning is occurring. ${ }^{52-54}$ The degree of change required in the health professional education system to fully embed patient safety is considerable and change is more likely to be successful when regulatory levers are used ${ }^{55}{ }^{56}$ in addition to more motivational approaches. Successfully building patient safety leadership at policy and regulatory levels (the first key action) can facilitate this kind of system-level action.

We have created a framework that captures these three developmental action areas and is aligned with the stages of patient safety curriculum implementation (figure 2). We agree with Jippes et al that the curriculum implementation process is "highly dynamic, non-linear,

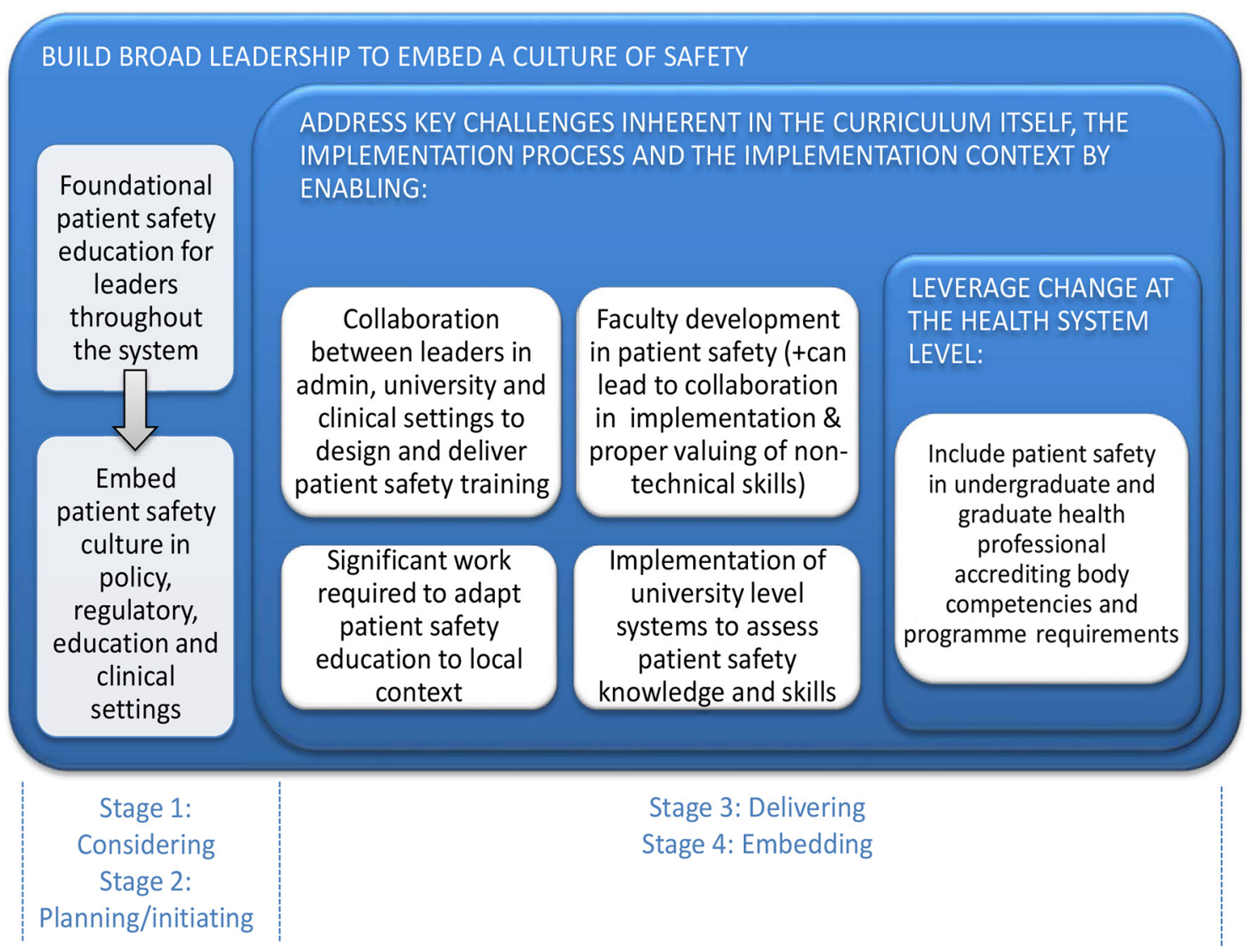

Figure 2 Framework for integrating patient safety science throughout health professional ieducation. 
and influenced by many factors' ${ }^{32}$ The three main elements in figure 2 should be seen as nested rather than linear in that building broad leadership is an important first step, but also needs to be ongoing in order to embed a culture of safety that can support integration of patient safety throughout health professional education.

Study limitations pertain largely to sample size. While we did achieve survey response rates of $42 \%$ and $52 \%$, our results are based on two, fairly small, respondent groups. We solicited and received information via email from non-responders to try to understand the nature of our non-response bias. These data confirm that it is unlikely non-responders are engaged in more patient safety curricular activities than the respondent group. However, we need to be cautious in generalising our results across low-income and middle-income countries. Neither the European nor African WHO regions participated in the study, and the African region has the highest proportion of low-income countries. The four regions that did participate in this study showed large variation in the proportion of countries able to identify a patient safety focal point. For instance, all 11 countries in the Southeast Asian region identified at least one focal point, but fewer than half of the 22 countries in the Western Pacific region were able to do so. Our sample was also not large enough to comment on differences in implementation between low-income and middle-income countries. Variation in patient safety curriculum implementation across low-income and middle-income countries is an area that requires further study.

Our findings on the scale of patient safety curriculum implementation point to the importance of further research in this area. Greater understanding of and attention to different roles and perspectives of individuals in policy versus university settings is needed. In our study and in other recent work, ${ }^{33}$ each of these stakeholders perceived the other as a barrier to achieving curricular change. Administrators perceived a lack of faculty support for curricular change. Faculty claimed that they were not being given sufficient infrastructure and resources to support curricular change. Case studies of resource-constrained jurisdictions that have had some success integrating patient safety into health professional education would be a valuable resource for countries trying to implement a patient safety curriculum.

Finally, there are several innovation diffusion questions that require further exploration ${ }^{37}$ that are particularly germane to patient safety curriculum implementation. For instance, how can innovations be adapted so they are perceived as advantageous and compatible with prevailing norms and values in a particular context? Is there a role for a central agency in curriculum implementation and, if so, what does that role look like? Who are the key opinion leaders in health professional education, and how should they be identified? What is their sphere of interpersonal influence? Lastly, what is the role of social and professional networks in enabling and promoting patient safety curriculum implementation across countries and regions? In-depth study of examples such as the Medical Councils Network of the WHO Southeast Asia region may enhance understanding of the potential effectiveness of these kinds of regional networks.

\section{CONCLUSIONS}

Ensuring that patients are protected from avoidable harm continues to be a priority for all healthcare systems around the world. An essential component of this goal is to address the challenges of embedding a patient safety science-based curricula throughout health professional education. Clear progress has been made in developing appropriate curricular resources. Attention must now focus on the implementation context and process, including developing leadership in patient safety at all levels in order to create an environment capable of meeting increasing needs for complex healthcare, in line with the UN Sustainable Development Goals.

Patient safety curricular change presents numerous challenges for low-income and middle-income countries and for developed countries including the need to target healthcare cultures dominated by authority gradients, incivility and very low levels of patient engagement. The implementation science and organisational psychology disciplines have much to offer as countries, and organisations and networks within and across their borders seek to move beyond treating patient safety as discrete curricular modules towards fully integrating patient safety into health professional education and training.

Correction notice This paper has been amended since it was published Online First. Owing to a scripting error, some of the publisher names in the references were replaced with 'BMJ Publishing Group'. This only affected the full text version, not the PDF. We have since corrected these errors and the correct publishers have been inserted into the references.

Contributors LG, ND-K and LJD made meaningful contributions to the conception (objectives) and design of the study; LG led the work on survey instrument design with input from ND-K and LJD. LG and ND-K determined sampling approach, and $L G$ was responsible for data collection and analysis. LG, ND-K and LJD participated in interpretation of data. LG drafted the initial manuscript including the PS curriculum implementation framework. ND-K and LJD critically revised the manuscript for intellectual content. LG, ND-K and LJD revised and approved the final version of the paper.

Competing interests None declared.

Patient consent Not applicable - patients are not part of this study. Ethics approval WHO Research Ethics Review Committee; York University REB. Provenance and peer review Not commissioned; externally peer reviewed. Data sharing statement № additional data are available.

Open Access This is an Open Access article distributed in accordance with the Creative Commons Attribution Non Commercial (CC BY-NC 4.0) license, which permits others to distribute, remix, adapt, build upon this work non-commercially, and license their derivative works on different terms, provided the original work is properly cited and the use is non-commercial. See: http://creativecommons.org/ licenses/by-nc/4.0/

(C) Article author(s) (or their employer(s) unless otherwise stated in the text of the article) 2017. All rights reserved. No commercial use is permitted unless otherwise expressly granted. 


\section{REFERENCES}

1. Lucian Leape Institute. Unmet needs:teaching Physicians to provide Safe Patient Care. Boston: National Patient Safety Foundation, 2010.

2. of HD, Careers MM. The New Curriculum for the Foundation years in Postgraduate Education and training. London, UK: Department of Health, 2007.

3. Hallmarks of quality and patient safety: recommended baccalaureate competencies and curricular guidelines to ensure high-quality and safe patient care. J Prof Nurs 2006;22:329-30.

4. Cronenwett L, Sherwood G, Barnsteiner J, et al. Quality and Safety Education for Nurses. Nurs Outlook 2007;55:122-31.

5. Frank JR, Brien S C. On behalf of TSCS. The Safety Competencies: Enhancing Patient Safety Across the Health Professions. Ottawa, ON: Canadian Patient Safety Institute, 2008.

6. Handler JA, Gillam M, Sanders AB, et al. Defining, identifying, and measuring error in emergency medicine. Acad Emerg Med 2000;7:1183-8.

7. Sandars J, Bax N, Mayer D, et al. Educating undergraduate medical students about patient safety: Priority areas for curriculum development. Med Teach 2007;29:60-1.

8. Walton M, Woodward $\mathrm{H}$, Van Staalduinen $\mathrm{S}$, et al. for and on behalf of the Expert Group convened by the World Alliance of Patient Safety, as Expert Lead for the Sub-Programme. The WHO patient safety curriculum guide for medical schools. BMJ Qual Saf 2010;19:542-6.

9. Walton MM, Shaw T, Barnet S, et al. Developing a national patient safety education framework for Australia. Qual Saf Health Care 2006;15:437-42.

10. Lempp $\mathrm{H}$, Seale $\mathrm{C}$. The hidden curriculum in undergraduate medical education: qualitative study of medical students' perceptions of teaching. BMJ 2004;329:770-3.

11. Wong BM, Etchells EE, Kuper A, et al. Teaching quality improvement and patient safety to trainees: a systematic review. Acad Med 2010;85:1425-39.

12. Kane JM. Patient safety education: overreported and still lacking. Acad Med 2010;85:1397-8

13. Alper E, Rosenberg E, O'Brien KE, et al. Patient Safety Education at U.S. and Canadian Medical Schools: Results from the 2006 Clerkship Directors in Internal Medicine Survey. Acad Med 2009;84:1672-6.

14. Ahmed M, Arora S, Tiew S, et al. Building a safer foundation: the Lessons Learnt patient safety training programme. BMJ Qual Saf 2014;23:78-86.

15. McCarthy SE, O'Boyle CA, O'Shaughnessy A, et al. Online patient safety education programme for junior doctors: is it worthwhile? Ir J Med Sci 2016;185:51-8.

16. Aboumatar HJ, Thompson D, Wu A, et al. Development and evaluation of a 3-day patient safety curriculum to advance knowledge, self-efficacy and system thinking among medical students. BMJ Qual Saf 2012;21:416-22.

17. Halbach JL, Sullivan LL. Teaching medical students about medical errors and patient safety: evaluation of a required curriculum. Acad Med 2005;80:600-6.

18. Madigosky WS, Headrick LA, Nelson K, et al. Changing and sustaining medical students' knowledge, skills, and attitudes about patient safety and medical fallibility. Acad Med 2006;81:94-101.

19. Gunderson AJ, Smith KM, Mayer DB, et al. Teaching medical students the art of medical error full disclosure: evaluation of a new curriculum. Teach Learn Med 2009;21:229-32.

20. Leung GK, Patil NG, Ip MS. Introducing patient safety to undergraduate medical students--a pilot program delivered by health care administrators. Med Teach 2010;32:e547-e551.

21. Miller CL, LaFramboise L. Student learning outcomes after integration of quality and safety education competencies into a senior-level critical care course. J Nurs Educ 2009;48:678-85.

22. Walton $M$, Jeffery $H$, Van Staalduinen $\mathrm{S}$, et al. When should students learn about ethics. professionalism and patient safety? Clin Teach 2013.

23. Walton MM, Elliott SL. Improving safety and quality: how can education help? Med J Aust 2006;184(10 Suppl):S60-S64 www.mja com.au.

24. Grimshaw J, Eccles M, Thomas R, et al. Toward evidencebased quality improvement. Evidence (and its limitations) of the effectiveness of guideline dissemination and implementation strategies 1966-1998. J Gen Intern Med 2006;21 Suppl 2:S14-20.

25. Bhattacharyya O, Reeves S, Zwarenstein M. What is Implementation Research?: rationale, concepts, and Practices. Res Soc Work Pract 2009;19:491-502.

26. Eliot TS. Between the idea and the reality falls the shadow. BMJ 1998;317:7156

27. Walton $\mathrm{M}$, Harrison $\mathrm{R}$, Burgess $\mathrm{A}$, et al. Workplace training for senior trainees: a systematic review and narrative synthesis of current approaches to promote patient safety. Postgrad Med J 2015:91:579-87.

28. Leotsakos A, Ardolino A, Cheung R, et al. Educating future leaders in patient safety. J Multidiscip Healthc 2014;7:381-8.

29. Fixsen DL, a BK, Naoom SF, et al. Res Soc Work Pract 2009;19:531-40.

30. Bank L, Jippes M, van Luijk S, et al. Specialty Training's Organizational Readiness for curriculum Change (STORC): development of a questionnaire in a Delphi study. BMC Med Educ 2015;15:127.

31. Bland CJ, Starnaman S, Wersal L, et al. Curricular change in medical schools: how to succeed. Acad Med 2000;75:575-94.

32. Jippes E, Van Luijk SJ, Pols J, et al. Facilitators and barriers to a nationwide implementation of competency-based postgraduate medical curricula: a qualitative study. Med Teach 2012;34:e589-e602.

33. Ali SK, Baig LA. Problems and issues in implementing innovative curriculum in the developing countries: the Pakistani experience. BMC Med Educ 2012;12:31.

34. World Health Organization. The Global Guardian of Public Health 2016 http://www.who.int/about/what-we-do/global-guardian-ofpublic-health.pdf (Accessed April 5, 2017).

35. World Health Organization. Definition of Region Groupings.. 2017 http://www.who.int/healthinfo/global_burden_disease/definition_ regions/en/ (Accessed April 5, 2017).

36. Pablos-Mendez A, Chunharas S, Lansang MA, et al. Knowledge translation in global health. Bull World Health Organ 2005;83:723.

37. Greenhalgh T, Robert G, Macfarlane F, et al. Diffusion of innovations in service organizations: systematic review and recommendations. Milbank Q 2004;82:581-629.

38. Huang GC, Newman LR, Tess AV, et al. Teaching patient safety: conference proceedings and consensus statements of the Millennium Conference 2009. Teach Learn Med 2011;23:172-8.

39. Klamen DL, Sanserino K, Skolnik P. Patient safety education: what was, what is, and what will be? Teach Learn Med 2013;25 Suppl $1: S 44-S 49$.

40. Kirch DG, Boysen PG. Changing the culture in medical education to teach patient safety. Health Aff 2010;29:1600-4.

41. Armitage G, Cracknell A, Forrest K, et al. Twelve tips for implementing a patient safety curriculum in an undergraduate programme in medicine. Med Teach 2011;33:535-40.

42. Cresswell K, Howe A, Steven A, et al. Patient safety in healthcare preregistration educational curricula: multiple case studybased investigations of eight medicine, nursing, pharmacy and physiotherapy university courses. BMJ Qual Saf 2013;22:843-54.

43. Wong BM, Levinson W, Shojania KG. Quality improvement in medical education: current state and future directions. Med Educ 2012;46:107-19.

44. Walton M, Barraclough B. Clinical supervisors: are they the key to making care safer? BMJ Qual Saf 2013;22:609-12.

45. Wong BM, Kuper A, Hollenberg E, et al. Sustaining quality improvement and patient safety training in graduate medical education: lessons from social theory. Acad Med 2013;88:1149-56.

46. Gandhi TK, Berwick DM, Shojania KG. Patient Safety at the Crossroads. JAMA 2016;315:1829-30.

47. Botwinick L, Bisognano M. Leadership Guide to Patient Safety. IHI Innovation Series White Paper. Cambridge, MA: Institute for Healthcare Improvement, 2006

48. WHO. WHO Patient Safety Curriculum Guide for Medical Schools. Switzerland: World Health Organization, 2009.

49. World Health Organization. 2011. Patient Safety Curriculum GuideMulti-professional Edition. Geneva.

50. Farley D, Zheng H, Rousi E, et al. Field Test of the World Health Organization Multi-Professional Patient Safety Curriculum Guide. PLoS One 2015;10:e0138510.

51. Lowry S. Strategies for implementing curriculum change. BMJ 1992;305:1482-5 http://www.pubmedcentral.nih.gov/articlerender. fcgi?artid=1884086\&tool=pmcentrez\&rendertype=abstract.

52. UK General Medical Council. Promoting excellence: standards for medical education and training.. 2015 http://www.gmc-uk.org/ education/standards.asp (Accessed April 4, 2017).

53. UK General Medical Council. Outcomes for graduates (Tomorrow's Doctors).. 2015 http://www.gmc-uk.org/Outcomes for graduates Jul_15_1216.pdf_61408029.pdf (Accessed April 4, 2017).

54. Australian Medical Council Limited. Standards for Assessment and Accreditation of Primary Medical Programs. 2012 http://www.amc.o rg.au/files/d0ffcecda9608cf49c66c93a79a4ad549638bea0_original. pdf (Accessed 4 Apr, 2017).

55. DiMaggio PJ, Powell WW. The Iron Cage Revisited: Institutional Isomorphism and Collective Rationality in Organizational Fields. Am Sociol Rev 1983;48:147-60 http://links.jstor.org.ezproxy.library. 
yorku.ca/sici?sici=0003-1224\%28198304\%2948\%3A2\%3C147\% 3ATICRII\%3E2.0.CO\%3B2-S.

56. Dixon-Woods M, McNicol S, Martin G. Ten challenges in improving quality in healthcare: lessons from the Health Foundation's programme evaluations and relevant literature. BMJ Qual Saf 2012;21:876-84. 